\title{
Precision engineering
}

\section{Ralph Delmdahl}

\author{
The excimer laser is synonymous with precision. Today it is enabling the production of integrated circuits and next- \\ generation displays, as well as new breakthroughs in eye surgery.
}

The UV excimer laser is one of the most precise industrial processing tools on the market and has many applications, of which eye surgery and semiconductor lithography are perhaps the best known. The great precision of excimer lasers results from the fact that the resolution achievable in laser materials processing is inversely related to the laser wavelength - the shorter the emission, the greater the achievable resolution. A UV excimer laser therefore offers considerable advantages over visible or infrared lasers for many applications.

Excimer lasers take their name from the term 'excited dimer' and are gas lasers that are electrically pumped using a high-voltage discharge. The gases commonly consist of an inert gas such as argon, krypton or xenon, and a reactive gas such as fluorine or chlorine. When electrically excited, the gas mixture temporarily forms excited states that emit UV light. The first laboratory demonstration of an excimer laser took place in 1970 at the Lebedev Physical Institute in Moscow. This laser was based on a xenon dimer and operated at a wavelength of $172 \mathrm{~nm}$.

Today, most industrial excimer lasers operate at wavelengths of $193 \mathrm{~nm}$ (ArF), $248 \mathrm{~nm}(\mathrm{KrF})$ and $308 \mathrm{~nm}(\mathrm{XeCl})$, corresponding to photon energies of $6.4 \mathrm{eV}$, $5.0 \mathrm{eV}$ and $4.0 \mathrm{eV}$, respectively. Depending on their wavelength of operation and substrate material, state-of-the-art excimer laser micromachining systems are capable of achieving lateral machining resolutions down to $1 \mu \mathrm{m}$, with vertical machining resolutions as small as $10 \mathrm{~nm}$ per laser pulse.

The first industrial excimer lasers in the early 1980s had organic polymer-based laser chambers, which fundamentally limited both the operational lifetime of the active gas and the chamber itself, owing to outgassing effects and material aging. The early 1990s saw the transition to metal/ceramic chambers entirely composed of special alloys and corrosionresistant high-density ceramics. This major technological breakthrough immediately boosted gas lifetimes and increased chamber lifetimes by an order of magnitude.

The mid-1990s saw the duty cycles and pulse frequencies of excimer lasers increase,

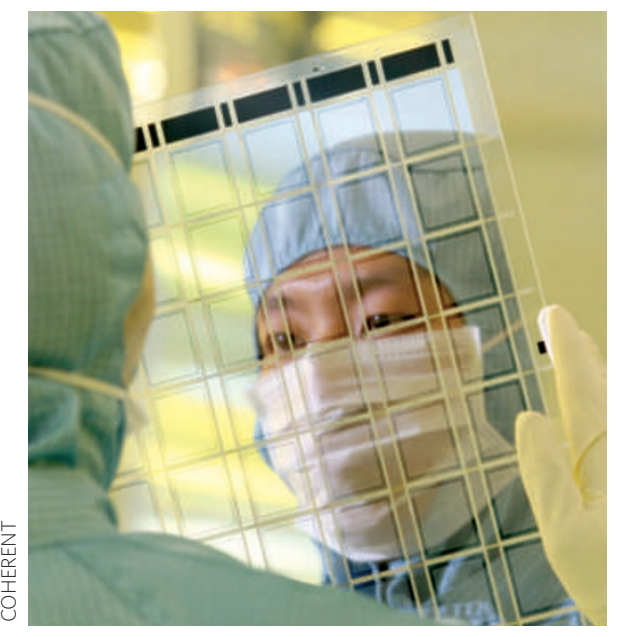

Excimer lasers are used in low-temperature poly-silicon annealing to transform thin layers of amorphous silicon into poly-crystalline silicon with largely enhanced electron mobility.

after which the operational lifetime of the thyratron switch - used to trigger highvoltage discharges in the laser gas - became the bottleneck for industrial use. The introduction of low-voltage semiconductor switching technology, combined with magnetic isolators and pulse transformers to achieve the required discharge voltages, eliminated the need for thyratron switches by the end of the 1990s. The replacement of the thyratron switch by its maintenancefree solid-state-based counterpart laid the foundation for the successful use of excimer lasers on the industrial production floor. In addition, the development of bandwidth-narrowing techniques that reduced excimer laser bandwidths from around $500 \mathrm{pm}$ to less than a thousandth of this value enabled excimer lasers to be used in photolithography.

Today, the two most prominent applications of excimer lasers are in semiconductor chip manufacturing and refractive eye surgery. Both of these rely on the deep-UV emission wavelength of $193 \mathrm{~nm}$, with semiconductor chip manufacturing capitalizing on the optical resolution of excimer lasers, and refractive eye surgery on its ability to ablate the cornea. For example, high repetition rate line-narrowed excimer laser models with $10 \mathrm{~mJ}$ per pulse, pulse frequencies of up to $6 \mathrm{kHz}$ and narrowed bandwidths of $0.35 \mathrm{pm}$ are used in advanced photolithography to produce computer chips with feature sizes of $45 \mathrm{~nm}$.

Another major industrial growth market for excimer lasers is low-temperature polysilicon annealing. Here, the $308 \mathrm{~nm}$ wavelength transforms 50 -nm-thin layers of amorphous silicon into high-quality polycrystalline silicon with greatly enhanced electron mobility, for use in flat-panel displays for mobile phones and flat-screen televisions.

In the low-temperature annealing of polysilicon, excimer lasers with UV output energies of over $1 \mathrm{~J}$ per pulse and output powers of $600 \mathrm{~W}$ are used to manufacture liquid-crystal and organic LED backplanes at a rate of $100 \mathrm{~cm}^{2} \mathrm{~s}^{-1}$.

The excimer laser is also used in the precision machining of medical devices made from polymers, glass or ceramics, and has a key role in the production of high-brightness LEDs, where its $193 \mathrm{~nm}$ and $248 \mathrm{~nm}$ wavelengths are crucial for the uniform lift-off of the GaN die from the sapphire substrate.

Ongoing miniaturization in microelectronics and the trend towards thin-film technologies demands increasing lateral resolution and selective machining. Functional structures and active layers are often only tens of nanometres thick and have to be annealed, patterned and removed in a selective manner without damaging underlying layers or substrates. Because excimer lasers provide the shortest wavelength of all laser technologies, they will continue to have a crucial role in many industries over the next decade.

In years to come we will see high-power applications of excimer lasers, with output energies reaching $2 \mathrm{~J}$ and output power levels of $1,000 \mathrm{~W}$ and above.

Ralph Delmdahl is product marketing manager

of the excimer business unit at Coherent,

Hans-Böckler-Str. 12, 37079 Göttingen, Germany.

e-mail: ralph.delmdahl@coherent.com 